\title{
Analysis by gas liquid chromatography of production of volatile fatty acids by anaerobic bacteria grown on solid medium
}

\author{
RJ WIGGINS, M WILKS, S TABAQCHALI \\ From the Department of Medical Microbiology, St Bartholomew's Hospital, London
}

SUMMARY Volatile fatty acids produced in Robertson's cooked meat medium by a range of clinically relevant anaerobes were compared by gas liquid chromatography with those produced in blood agar. The same volatile fatty acid profiles were obtained in both media, although the concentration of acids was lower in blood agar. We conclude that detection of volatile fatty acids from a pure culture of an organism on solid medium is practicable and offers advantages over the conventional technique.

The value of gas liquid chromatography in identifying anaerobic bacteria by detecting the short chain fatty acids which they produce has been clearly established. ${ }^{\prime 2}$ A profile of the volatile fatty acids produced is readily obtained and is a useful adjunct to biochemical tests in the formal identification of anaerobic isolates.

Conventionally, a pure culture of the organism is first obtained on solid medium and subcultured into a liquid medium containing one or more carbohydrates. The liquid medium is then incubated for 48 hours and an extract analysed by gas liquid chromatography. If the same short chain fatty acids are present in similar amounts in extracts prepared from the initial pure culture of the organism on solid medium this would obviate the need to incubate broth cultures, consequently saving time and culture medium. A method has recently been described in which production of volatile fatty acid by Clostridium difficile was compared in primary isolation medium and in cooked meat broth. ${ }^{3}$ The same volatile fatty acids were found in similar quantities in both media.

We used a similar method to examine the production of volatile fatty acids by a range of commonly encountered anaerobic bacteria grown on brucella base blood agar. We compared the results with those obtained from Robertson's cooked meat medium containing carbohydrates. Some anaerobic isolates produce visible growth on solid medium after 24 hours of incubation and we therefore examined pro-

$$
\text { Accepted for publication } 3 \text { May } 1985
$$

duction of volatile fatty acid in both solid and liquid media after 24 hours of incubation.

\section{Material and methods}

\section{BACTERIA}

Previously identified routine clinical isolates of the following species were used: twenty eight $\mathrm{Bac}$ teroides fragilis; 16 other isolates of the Bact fragilis group; six Clostridium perfringens, 10 Clostridium difficile, 10 other clostridia; seven peptococci, eight peptostreptococci.

\section{MEDIA}

Each organism was inoculated on to two blood agar plates (brucella base (Difco): $7 \%$ horse blood; vitamin $\mathrm{K} /$ haemin $10 \mathrm{mg} / \mathrm{l}$ ) and into two bottles of cooked meat medium (Robertson's cooked meat; glucose $0.5 \%$; maltose $0.1 \%$; cellobiose $0.1 \%$, Southern Group Laboratories).

\section{CULTURE}

The blood plates were incubated at $37^{\circ} \mathrm{C}$ in an anaerobic chamber $(80 \%$ nitrogen, $10 \%$ hydrogen, $10 \%$ carbon dioxide) and the cooked meat medium in a bijou bottle with a screw cap in an incubator at $37^{\circ} \mathrm{C}$, without using an anaerobic jar. Extracts of each kind of medium were analysed by gas liquid chromatography after incubation for 24 and 48 hours.

PROCEDURE FOR EXTRACTION

Agar was removed from the well area of plates using 
a cork borer $1 \mathrm{~cm}$ in diameter. Both media were then acidified using $0.3 \mathrm{ml} 50 \%$ sulphuric acid. An equal volume of diethyl ether was added to $1.5 \mathrm{ml}$ of cooked meat medium supernatant $1.5 \mathrm{ml}$ agar in a glass tube with a screw top, and or, vortex mixed for 30 seconds and two minutes, respectively. Solvent and aqueous phases were separated by centrifugation for one minute.

\section{CHROMATOGRAPHY}

A Perkin-Elmer Sigma 1 chromatograph fitted with a flame ionisation detector and linked to a PerkinElmer Sigma 10 computing integrator was used. A $6 \mathrm{ft} \times 1 / 4$ inch column packed with $20 \%$ neopentyl glycol adipate (Pye-Unicam) on acid washed celite was used. The injection temperature was $160^{\circ} \mathrm{C}$ and the oven temperature $141^{\circ} \mathrm{C}$. The nitrogen carrier gas flow rate was $40 \mathrm{ml} / \mathrm{min}$. The injection volume was $1 \mu$ l.

\section{Results}

Extracts prepared from uninoculated media after incubation were tested for volatile fatty acids by gas liquid chromatography. Volatile fatty acids were not detected in any samples of blood agar tested. Small amounts of acetic acid were found in extracts prepared from cooked meat medium.

The concentrations of volatile fatty acids produced by most organisms in either medium after 24 hours of incubation were generally insufficient to be useful in identification. The strains which did produce adequate concentrations of volatile fatty acids after 24 hours (mostly clostridia) were too few in number to justify reducing the conventional time for incubation.

Although we analysed the volatile fatty acid content of agar taken from the well area of plates, volatile fatty acids can be detected in agar taken from other areas, and the use of pure cultures is therefore imperative.

Figs 1-4 show the production of volatile fatty acids by Bact fragilis, $C$ perfringens, $C$ difficile, and anaerobic Gram positive cocci, respectively. The table shows the production of volatile fatty acids by the other less commonly isolated species.

\section{Discussion}

The production of volatile fatty acids by anaerobes as metabolic end products has been exploited for the rapid presumptive diagnosis of anaerobic infections ${ }^{4}$ and for the identification of organisms, ${ }^{2}$ as volatile fatty acids are easily detected by gas liquid chromatography. Although this procedure permits presumptive diagnosis of anaerobic infections within 30 minutes of receipt of the specimen, identification of isolates may take several days.

Methods for rapid presumptive identification ${ }^{3}$ and for rapid definitive identifications of $C$ difficile, using gas liquid chromatography, have recently been described. Volatile fatty acids have been detected in extracts prepared from standard ${ }^{3}$ and modified ${ }^{5}$ selective media and it is suggested that this obviates the need to incubate broth cultures with a subsequent delay in reporting of $24-48$ hours.

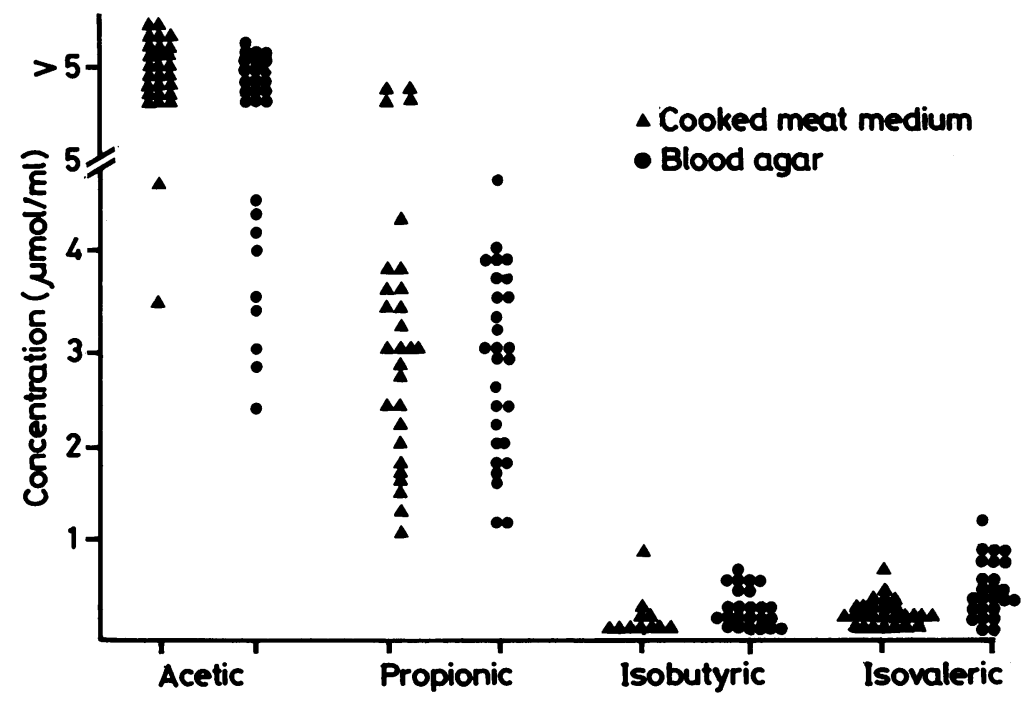

Fig. 1 Production of volatile fatty acid by Bacteroides fragilis 


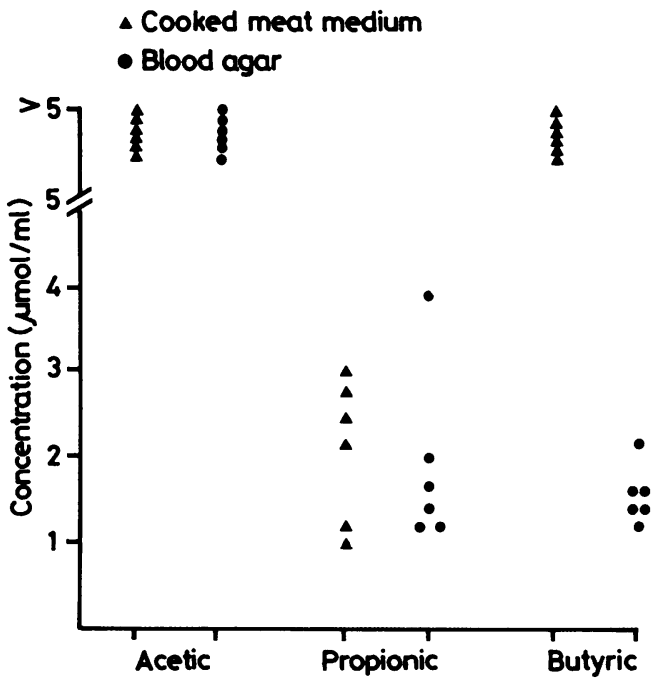

Fig. 2 Production of volatile fatty acid by Clostridium perfringens

We found that the same volatile fatty acids are produced in blood agar as in cooked meat medium by Bact fragilis, other members of the Bact fragilis group, $C$ difficile, a range of other clostridia, and anaerobic Gram positive cocci. The concentrations of volatile fatty acids in blood agar were generally less than those in the corresponding cooked meat

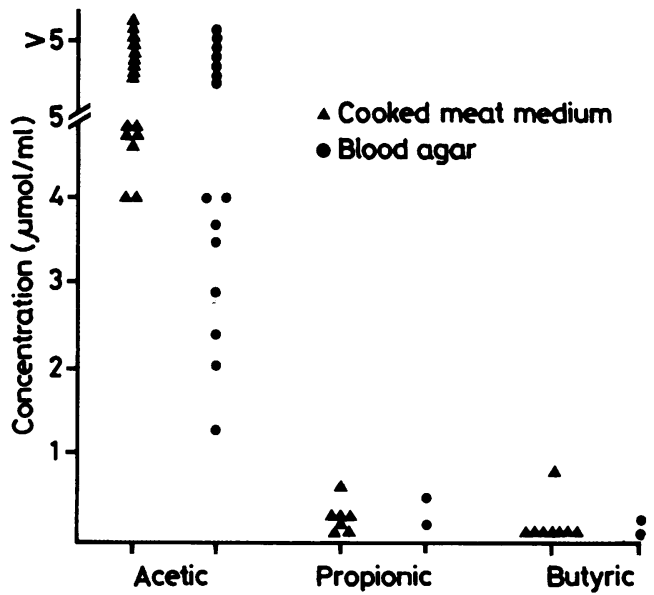

Fig. 4 Production of volatile fatty acid by anaerobic gram positive cocci

medium, but this did not create any difficulties in interpreting the profiles produced. Identical volatile fatty acid profiles were not obtained in both media for some strains of species which produce only small amounts $(<1 \mu \mathrm{g} / \mathrm{ml})$ of some volatile fatty acids. Minor discrepancies in results such as these do not, however, diminish the usefulness of gas liquid chromatography for identifying such organisms.

We conclude that estimating volatile fatty acids

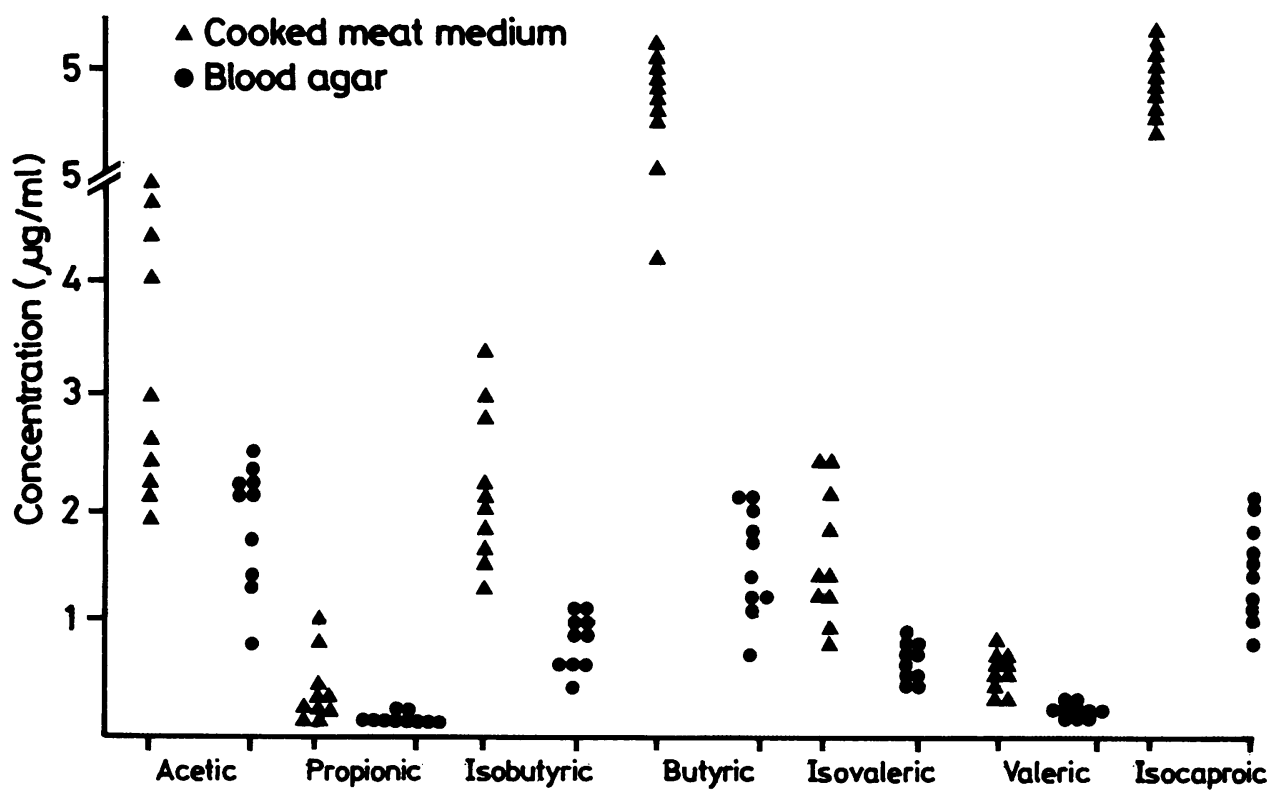

Fig. 3 Production of volatile fatty acid by Clostridium difficile 
Table

\begin{tabular}{|c|c|c|}
\hline Organism $(n=)$ & $\begin{array}{l}\text { Volatile fatty acids produced in: } \\
\text { Cooked meat medium }\end{array}$ & Blood agar \\
\hline 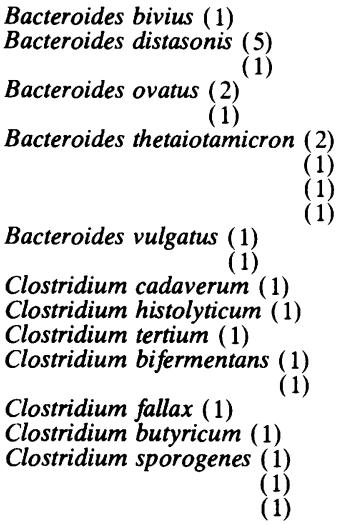 & $\begin{array}{l}\text { A } \\
\text { A P } \\
\text { A P iv } \\
\text { A P } \\
\text { A P iv } \\
\text { A P ib iv } \\
\text { A p } \\
\text { A p iv } \\
\text { A p ib } \\
\text { A P } \\
\text { A P } \\
\text { A P B } \\
\text { A } \\
\text { A B } \\
\text { A } \\
\text { A } p \text { iv } \\
\text { A B } \\
\text { A B } \\
\text { A P IB B IV IC } \\
\text { A P IB B IV V IC } \\
\text { A P IB B IV IC }\end{array}$ & 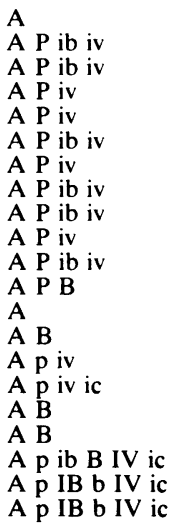 \\
\hline
\end{tabular}

Upper case letters: $>1 \mathrm{ug} / \mathrm{ml}$; lower case letters: $<1 \mathrm{ug} / \mathrm{ml}$.

$\mathrm{A}$ : acetic acid; $\mathrm{P} / \mathrm{p}$ : propionic acid; IB/ib: isobutyric acid; $\mathrm{B} / \mathrm{b}$ : butyric acid; IV/iv: isovaleric acid; $\mathrm{V} / \mathrm{v}$ : valeric acid; IC/ic: isocaproic acid.

from a pure culture on solid medium produces results equivalent to the conventional technique and saves both time and media.

\section{References}

' Moore WEC. Relationships of metabolic products to taxonomy of anaerobic bacteria. Int J System Bact 1970;20:535-8.

${ }^{2}$ Holdeman LV, Cato EP, Moore WEC. Anaerobe laboratory manual. 4th ed. Blacksburg: Virginia Polytechnic Institute and State University Anaerobe Laboratory, 1977.

${ }^{3}$ Makin T. Rapid identification of Clostridium difficile by direct detection of volatile organic acids from primary isolation media. J Clin Pathol 1984;37:711-2.

${ }^{4}$ Phillips KD, Tearle PV, Willis AT. Rapid diagnosis of anaerobic infections by gas liquid chromatography of clinical material. $J$ Clin Pathol 1976; 29:428-32.

${ }^{5}$ Levett PN, Phillips KD. Gas chromatographic identification of Clostridium difficile and detection of cytotoxin from a modified selective medium. J Clin Pathol 1985;38:82-5.

Requests for reprints to: Dr RJ Wiggins, Department of Medical Microbiology, St Bartholomew's Hospital, London EC1A 7BE, England. 